\title{
Left ventricular encasement after pneumonectomy: Case report
}

\author{
Sietske A. Smulders, MD, ${ }^{a}$ Johan T. Marcus, PhD, ${ }^{\mathrm{b}}$ C. Tji-Joong Gan, MD, ${ }^{\mathrm{c}}$ Thomas G. Sutedja, MD, PhD, ${ }^{\mathrm{c}}$
} Frank W. J. M. Smeenk, MD, PhD, and Anton Vonk-Noordegraaf, MD, PhD, ${ }^{c}$ Amsterdam, The Netherlands

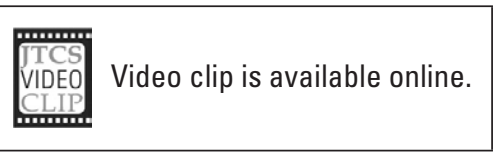

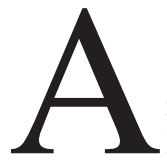

53-year-old man with a history of hypertension and left-sided pneumonectomy 7 years previously was referred to our hospital. He reported dizziness and had collapsed twice while digging. No other symptoms were present, especially no dyspnea. There were no signs of airway obstruction (forced vital capacity $51 \%$ and forced expiratory volume in 1 second $47 \%$ of predicted), and carbon monoxide diffusion capacity was normal (106\% of predicted when corrected for alveolar volume). Maximum exercise test results were normal for a patient who had undergone pneumonectomy, with maximum weight of $62 \%$ and maximum oxygen consumption of $77 \%$ of predicted. Arterial blood gas analysis showed normal ventilation. Echocardiography showed normal right ventricular and left ventricular (LV) functions and slight LV free wall flattening. Finally, dynamic cardiac magnetic resonance imaging (MRI) was performed to study the influence of increasing abdominal pressure on the right ventricular and LV function on a beat-per-beat basis. During MRI imaging, we asked the patient to perform a Valsalva maneuver while shortaxis cinematic images were made (Figure 1).As is clear from Figure 1, the position of the heart in this patient had shifted in the years after his pneumonectomy, and the LV free wall was repositioned all the way on top of the paralyzed left hemidiaphragm. During the Valsalva maneuver, intra-abdominal pressure increases, which probably hampered LV filling in this patient by a direct transmission of the intra-abdominal pressure to the LV free wall. Note that in the normal thorax the LV free wall is adjacent to the left lung, free from contact with the diaphragm. We also asked the patient to lift both his legs, mimicking a digging position, and to hold his breath for approximately 10 seconds while lying supine in the MRI scanner. The real-time short-axis cinematic images (Figure 2 and Video)

From the Department of Pulmonology, Catharina Hospital, Eindhoven, The Netherlands, a and the Departments of Physics and Medical Technology ${ }^{\mathrm{b}}$ and Pulmonology, ${ }^{\mathrm{c}}$ VU University Medical Center, Amsterdam, The Netherlands.

Received for publication March 13, 2006; accepted for publication April 5, 2006.

Address for reprints: A. Vonk-Noordegraaf, MD, PhD, VUMC, Department of Pulmonology, De Boelelaan 1117, 1081 HV Amsterdam, The Netherlands (E-mail: a.vonk@vumc.nl).

J Thorac Cardiovasc Surg 2006;132:e23-4

$0022-5223 / \$ 32.00$

Copyright (C) 2006 by The American Association for Thoracic Surgery doi:10.1016/j.jtcvs.2006.04.014

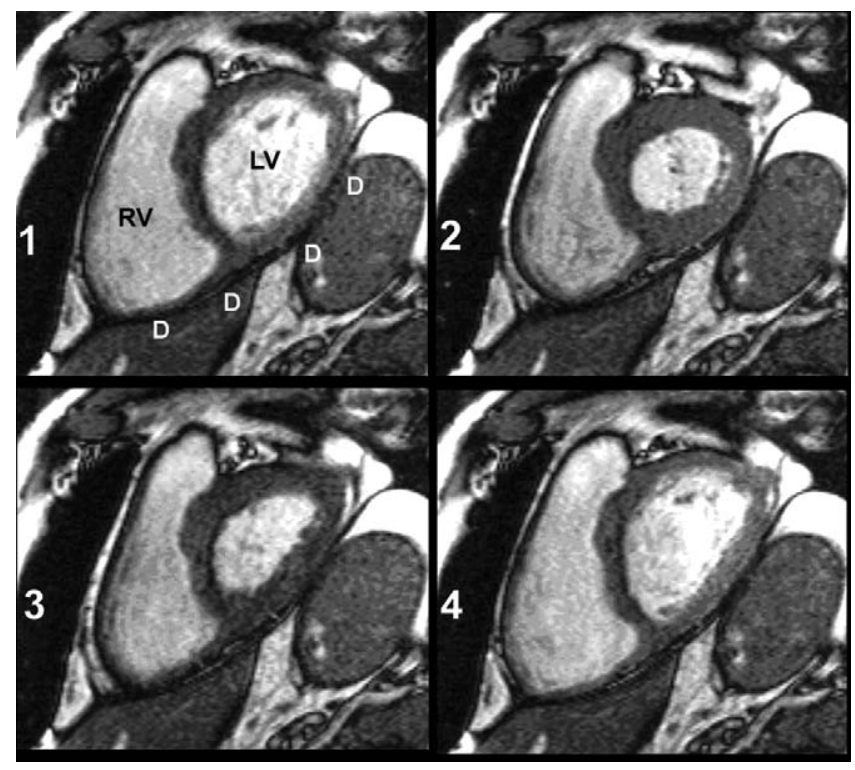

Figure 1. Ventricular short-axis images. Numbers 1 through 4 are consecutive images during Valsalva maneuver. Notice that LV wall flattening is most clearly seen in 3. 1 , Start of systole at $R$ wave; 2 , mid systole; 3 , early diastolic phase; 4 , late diastolic phase; $D$, left hemidiaphragm; RV, right ventricle.

showed a progressive LV free wall flattening, leading to a decrease in LV cross-sectional area, as shown in Figure 2 and the Video. These changes of the LV cross-sectional area at the midventricular level probably represent the LV volume changes. Thus the LV end-diastolic volume and stroke volume decreased as a result of the shifted position of the LV on top of the paralyzed hemidiaphragm which seemed to worsen its compliance. LV filling was further deteriorated during breath-holding and leg-tilting maneuvers. In retrospect, it appears that during pneumonectomy the phrenic nerve was severed with the intention of causing the postpneumonectomy space to fill faster postoperatively, a method that is currently considered controversial. ${ }^{1}$ Although we initially proposed surgical plication of the diaphragm as a possible solution, the low frequency of collapse (twice), uncertainty of the outcome of surgery, and the assurance of the patient after being informed about the reason of his collapse ultimately were factors in opting not to pursue a surgical solution.

This case report presents an unusual long-term complication of pneumonectomy, which was seen during real-time dynamic cardiac MRI imaging. Dynamic MRI provides an excellent tool to analyze the complications caused by the relocation of the 


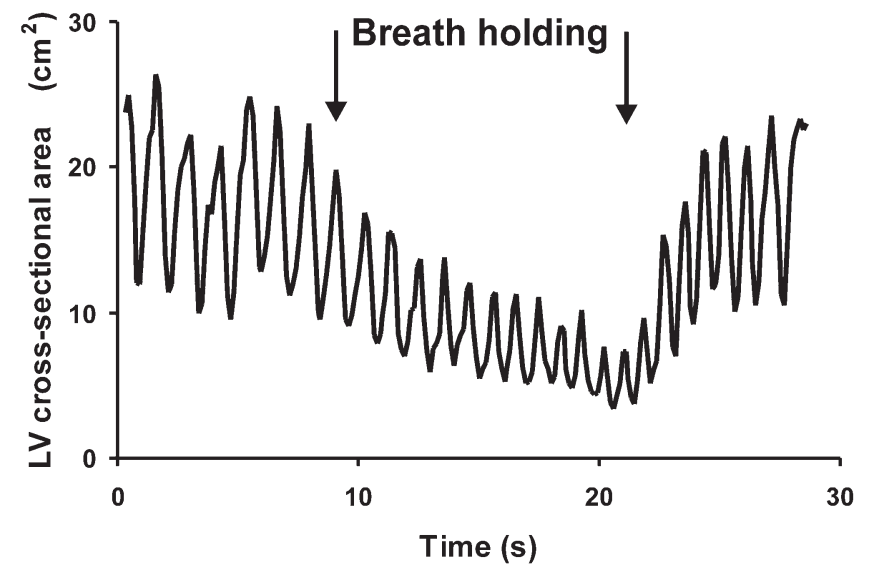

Figure 2. LV short-axis cross-sectional area (in square centimeters) at midventricular level plotted against time (in seconds). After about 8 seconds, influence of breath-holding maneuver with lifting both legs in supine position is clearly seen. LV crosssectional area comes back to initial values during normal breathing with both legs stretched and relaxed again. heart and large vessels in the patient who has undergone pneumonectomy.

\section{Reference}

1. Fell SC. Special article: a brief history of pneumonectomy. 1999. Chest Surg Clin North Am. 2002;12:541-63. 